Research Article

\title{
Guidance Law with Desired Impact Time and FOV Constrained for Antiship Missiles Based on Equivalent Sliding Mode Control
}

\author{
Shuangxi Liu $\mathbb{D}^{1}$, Binbin Yan $\mathbb{D},{ }^{2}$ Tong Zhang $\mathbb{D}^{1},{ }^{1}$ Pei Dai $\mathbb{D},{ }^{2,3}$ and Jie Yan ${ }^{1}$ \\ ${ }^{1}$ Unmanned System Research Institute, Northwestern Polytechnical University, Xi'an 710072, China \\ ${ }^{2}$ School of Astronautics, Northwestern Polytechnical University, Xi'an 710072, China \\ ${ }^{3}$ School of Aerospace Science and Technology, Xidian University, Xi'an 710126, China \\ Correspondence should be addressed to Tong Zhang; zhangtong@nwpu.edu.cn
}

Received 1 April 2021; Revised 13 July 2021; Accepted 2 September 2021; Published 25 September 2021

Academic Editor: Antonio Concilio

Copyright ( 2021 Shuangxi Liu et al. This is an open access article distributed under the Creative Commons Attribution License, which permits unrestricted use, distribution, and reproduction in any medium, provided the original work is properly cited.

\begin{abstract}
Impact time control guidance (ITCG) is an important approach to achieve saturation attack on targets. With the increasing complexity of warfare requirements for missiles, an ITCG with field-of-view (FOV) constrained for antiship missiles is proposed based on equivalent sliding mode control. Firstly, in view of the accuracy of the calculation of remaining impact time for guidance law, the large initial lead angle is taken into consideration in the estimation of remaining flying time in which there is no need for the assumption of small angle approximation. Besides, for the sake of promoting the practical application value of the proposed guidance law, FOV is considered so that it can satisfy the actual working performance of the seeker. Then, combined with the concept of predicted interception point (PIP), the proposed guidance law is applied to attack a moving target. Numerical analysis is carried out for different initial lead angles, various impact time, different methods of estimating remaining flying time, and cooperative attack conditions. Compared with proportion navigation guidance (PNG), the feasibility and effectiveness of the guidance law are verified. Simulation results demonstrate that the proposed guidance law can guarantee the constraints of both impact time and FOV effectively.
\end{abstract}

\section{Introduction}

In recent years, in the development of weapon equipment, antiship operations are faced with an unprecedented environment and presenting unprecedented new characteristics. As an irreplaceable precision guidance weapon in sea warfare, antiship missiles have played an extremely important role in previous naval battles since their creation. With the continuous improvement of modern warfare requirements for the damage effect of missiles, traditional guidance methods whose main design purpose is aimed at attacking the target have been difficult to accommodate the specific needs of modern warfare. As a result, researches on guidance law design with various constraints to improve the survivability and attack performance of missiles have been widely taken into consideration. For instance, in order to achieve the tactical effect of such saturate attack, it is necessary to realize the impact time control guidance (ITCG) for missiles so that each missile can independently or cooperate with each other to complete the attacking mission according to the specified impact time $[1,2]$.

In [3], Kim et al. firstly put forward the guidance problem of ITCG. Then, researchers from various countries based on different guidance schemes and control theories have achieved many research results on ITCG [4-13]. The accuracy of estimating the remaining flying time is the basis of ITCG. In order to obtain the remaining flight time as accurate as possible, many experiments tried to improve the estimation accuracy by compensating for the estimation error caused by the ballistic curvature. The main existing algorithms are included in application of recursive algorithm [4-6], polynomial approximate [7-9], numerical integration [10], and solution of trajectory [11-13]. In [4], a recursive algorithm is proposed to compensate for the remaining 
flight time estimation error caused by the flight lead angle of the missile, so as to obtain a more accurate remaining flight time. Based on the concept of polynomial approximate, a guidance law is proposed to obtain the remaining flight time by constructing an approximate polynomial of the ballistic trajectory with respect to the lead angle in [8]. By obtaining the ballistic trajectory between missile and attack point through numerical integration, Ref. [10] obtains the remaining flight time indirectly. Moreover, in order to promote the damage on targets, the impact angle problem is taken into consideration in $[11,12]$, in which the remaining flight time is estimated based on the assumption of small angle approximation.

In addition, a variety of suitable remaining flight time estimation methods for different specific conditions have been conducted in [14-17]. A remaining flight time estimation filter is proposed for antiship missiles in [15], by applying Kalman filter theory; the remaining flight time estimation error caused by target motion and seeker noise interference is solved. In [16], with impact angle constrained, a logic conversion method for estimating remaining flight time for large lead angles has arisen. By approximating the trigonometric function to estimate the remaining flight time through McLaughlin polynomials, Ref [17] puts forward a guidance scheme based on the history of guidance instruction time.

Besides, in recent years, cooperative guidance law has been largely studied in view of with the constraint of impact time [18]. Through quantitative advantages, multiple missiles can exchange information each other so that they can complete a saturation attack on target from different directions, which is a handy way of foiling missile defense systems. In [19], a sufficient condition in terms of the properties of the arbitrary time-varying communication topologies is established to achieve the consensus of impact time. In [20], a cooperative guidance law employs the available measurements of relative impact time error as the feedback information to achieve the consensus of impact time among missiles.

In summary, it is important to point out that the core of ITCG is to estimate the remaining flying time of missiles, which has enormous influence on the performance of corresponding guidance laws and the attack accuracy. The research in regard to the estimation of remaining flying time can be divided into two categories. The first one is based on the concept of trajectory tracking so that the problem of estimating the remaining flying time can be converted into the tracking of missile's trajectory indirectly. However, it is of great difficulty to achieve the ideal tracking of the trajectory. The second one is to calculate the remaining flying time through the specific method. Nonetheless, as for the mentioned guidance schemes, there are some disadvantages in the estimation of the remaining flight time at a large initial lead angle or in specific attack time control guidance law design. On the one hand, the estimation accuracy of the remaining flight time based on the assumption of small angle approximation is insufficient under the large lead angle, which will consume a lot of computing resources and time. On the other hand, the moving targets are not being taken into consideration so they are lacking of application value. Most importantly, these guidance schemes only consider the constraint of impact time blindly and ignore the constraint of seeker's FOV, which may not satisfy the requirements of practical application scenarios.

Therefore, motivated by previous discussion, for antiship missiles attacking a target with impact time constrained, we propose a guidance scheme with both desired impact time and FOV constrained. The main contributions can be summarized as follows:

(1) In view of the actual working characteristics of the seeker, an ITCG law is proposed under the premise of satisfying the constraint of the seeker's FOV, which improves the practical application value of the proposed guidance law

(2) The large initial lead angle is taken into consideration in the process of calculating the remaining flying time so that there is no need to adopt the concept of the assumption of small angle approximation, which results in the fact that the calculation accuracy of impact time can be guaranteed

(3) Based on the equivalent sliding mode control algorithm, the guidance law is designed by selecting the error of impact time. Meanwhile, by utilizing the concept of PIP, the proposed guidance law can be expanded to moving targets

The remainder of this paper is organized as follows: Section 2 addresses the problem of an antiship missile attacks a target in the longitudinal plane, and the design process of the guidance law is shown in Section 3. Simulation studies are presented in Section 4, and conclusions are drawn in Section 5.

\section{Problem Statement}

Take into account the antiship combat scenario for example where the speed change of target can be approximately ignored so that the target can be regarded to be stationary due to the particularity of the antiship combat scenario. For the convenience of guidance design and analysis, it is assumed that a missile attacks a stationary target in the longitudinal plane as is shown in Figure 1. Thus, the kinematic relationship between them in the LOS coordinate system can be described as

$$
\begin{aligned}
\dot{R} & =-V \cos \eta, \\
R \dot{\gamma} & =-\sin \eta, \\
\dot{\theta} & =\frac{a}{V}, \\
\eta & =\theta-\gamma .
\end{aligned}
$$

In order to avoid the problem that the missile loses its target caused by the maneuvering of the missile, it is necessary to consider the constraint of the seeker's FOV when designing the guidance law. Then, in order to conduct the guidance law with desired impact time and FOV constrained 


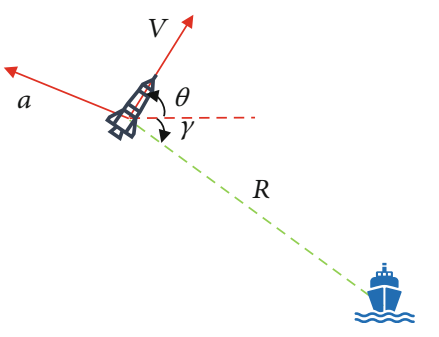

FIGURE 1: Plane attack diagram of missile.

at the same time, some premise assumptions need to be made in advance.

Assumption 1. Both the missile and target are regarded as ideal particle mass points, ignoring the influence of their own shape.

Assumption 2. The actuators of missile are all in an ideal working state, which means that there is no time delay phenomenon.

Assumption 3. The influence of the rotation of the earth and the external environment on missile and target are ignored.

It is noted that the angle between the longitudinal axis of missile and the direction of speed is small enough; the limited FOV can be approximately equivalent to the leading angle of missile $[21,22]$. Therefore, subject to Assumptions $1-3$, the objective of guidance law is to design the acceleration instruction $a$ to achieve the goal of attacking target precisely with impact time and FOV constrained. That is,

$$
\left\{\begin{array}{l}
\lim _{t \longrightarrow t_{d}} R \longrightarrow 0, \\
\lim _{t \longrightarrow t_{d}}\left(t_{\mathrm{im}}-t_{d}\right) \longrightarrow 0, \\
|\eta(t)| \leq \eta_{\max }, \quad t \in\left[0, t_{d}\right]
\end{array}\right.
$$

where $t_{d}$ is desired impact time, $t_{\text {im }}$ is actual impact time, and $\eta_{\max }$ is the upper boundary of FOV.

The specific design process of the acceleration instruction will be given in the next section.

\section{Main Results}

3.1. Estimation of Impact Time. In the whole process of terminal guidance, the impact time can be expressed as

$$
t_{\mathrm{im}}=t_{\mathrm{el}}+t_{\mathrm{go}}
$$

where $t_{\mathrm{el}}$ represents elapsed time after launch and $t_{\mathrm{go}}$ stands for remaining flying time. In order to satisfy the requirement of attack with desired impact time constrained, it is essential to calculate impact time $t_{\text {im }}$ accurately. Thus, the task is transformed to pursue a proper approach to estimate $t_{\mathrm{go}}$ accurately.

It is of enormous importance in actual combat scenarios to calculate $t_{\text {go }}$ precisely, especially for specific combat scenarios. Until now, there are main two methods to estimate $t_{\text {go }}$.

$$
t_{\mathrm{go}} \approx \widehat{t}_{\mathrm{go}}=\frac{R}{V_{c}}=-\frac{R}{V_{r}}=-\frac{R}{\dot{R}},
$$

where $V_{r}=\dot{R}$ is relative velocity between missile and target and $V_{c}=-V_{r}$ is closing velocity.

$$
\begin{aligned}
& t_{\mathrm{go}} \approx \widehat{t}_{\mathrm{go}}=\frac{R}{V} \gamma, \\
& r=1+\frac{\eta^{2}}{4 N-2}=1+\frac{(\theta-\gamma)^{2}}{4 N-2},
\end{aligned}
$$

where $Y$ is an adjustment factor composed of ballistic inclination angle $\theta$ and LOS angle $\gamma$. $N$ stands for the navigation coefficient which is usually selected as $2 \sim 6$.

For Equation (7), it is calculated by the ratio of relative range and velocity where only $R, \dot{R}$ defined in the LOS coordinate system are used so that the inner dynamics of missile cannot be reflected intuitively. Equation (8) is established on the basis of Equation (7) in which the physical quantities related to missile's own movement $V, \theta$ are considered. Most importantly, relying on adjustment factor $\gamma, t_{\mathrm{go}}$ can be amended according to the actual attack scenario. For instance, when a missile moves straightly towards a stationary target, $\eta=0$ holds, which demonstrates that $V=V_{c}$ so that the values of $t_{\text {go }}$ are the same based on the above two methods. On the other hand, in Equation (8), when a missile moves away from the target, there exists a heading error exactly. The larger the heading error becomes, the greater the adjustment factor $\boldsymbol{r}$ grows, and hence, the larger the $t_{\mathrm{go}}$ is. Furthermore, Equation (7) only depends on the relative velocity so that the heading error is ignored, which is not in line with practical combat scenarios.

However, it is necessary to note that Equation (8) holds on the basis of small-angle assumption (which requires small values for $\gamma, \theta$ ). In the practical process of terminal guidance, the initial position information of missile and target cannot be guaranteed that the initial values of $\gamma, \theta$ are small. As a result, inspired by [2], an improved estimation method of $t_{\mathrm{go}}$ is chosen as follows:

$$
\begin{aligned}
t_{\mathrm{go}} & \approx \widehat{t}_{\mathrm{go}}=\frac{R}{V_{M}} \Psi, \\
\Psi & =1+\frac{\sin ^{2} \eta}{4 N-2}=1+\frac{\sin ^{2}(\theta-\gamma)}{4 N-2},
\end{aligned}
$$

where $\Psi$ is an adjustment factor working the same as $\mathcal{Y}$ in Equation (8), which is relying on the heading error without considering small-angle assumption. Compared with $r$, the improved adjustment factor $\Psi$ has a greater application value to eliminate the heading error of the initial position information to estimate $t_{\text {go }}$ more accurately. 
3.2. Acceleration Instruction Design. Define a sliding mode variable $s$ as follows:

$$
s=t_{\mathrm{im}}-t_{d}=t_{\mathrm{el}}+t_{\mathrm{go}}-t_{d} .
$$

The derivative of Equation (10) can be presented as

$$
\dot{s}=\dot{t}_{\mathrm{el}}+\dot{t}_{\mathrm{go}}-\dot{t}_{d}=1+\dot{t}_{\mathrm{go}}
$$

Substituting Equation (9) into Equation (11), it can be conducted

$$
\begin{aligned}
\dot{s}=1 & +\dot{t}_{\mathrm{go}}=1+\frac{\dot{R}}{V}\left[1+\frac{\sin ^{2}(\theta-\gamma)}{4 N-2}\right] \\
& +\frac{R}{V(4 N-2)}[2 \sin (\theta-\gamma) \cos (\theta-\gamma)(\dot{\theta}-\dot{\gamma})] .
\end{aligned}
$$

Then, combining Equations (1)-(4) with Equation (12), one can yield

$$
\begin{aligned}
\dot{s}= & 1+\frac{-V \cos \eta}{V}\left[1+\frac{\sin ^{2}(\theta-\gamma)}{4 N-2}\right] \\
& +\frac{R}{V(4 N-2)}[2 \sin (\theta-\gamma) \cos (\theta-\gamma)(a / V+\dot{\gamma})] \\
= & 1-\cos (\theta-\gamma)\left[1+\frac{\sin ^{2}(\theta-\gamma)}{4 N-2}\right]+\frac{R}{V(2 N-1)} \\
& \cdot\left[\sin (\theta-\gamma) \cos (\theta-\gamma)\left(\frac{a}{V}-\dot{\gamma}\right)\right] .
\end{aligned}
$$

Based on the general form of the equivalent sliding mode control algorithm, acceleration instruction can be expressed into two parts:

$$
a=a_{\mathrm{eq}}+a_{\mathrm{disc}}
$$

where $a_{\mathrm{eq}}$ is equivalent control instruction in order to ensure system states reach on the selected sliding mode surface and $a_{\text {disc }}$ is discontinuous control instruction to make sure that when the system comes to the sliding mode surface, system states will stay on it all the time.

Setting $\dot{s}=0$, thus, $a_{\text {eq }}$ can be described as

$$
a_{\mathrm{eq}}=\frac{V^{2}[\cos \eta-1](2 N-1)}{R \sin \eta \cos \eta}+\frac{V^{2} \sin \eta}{2 R}+V \dot{\gamma} .
$$

Letting $a_{\text {disc }}=-k s \cdot \operatorname{sgn}^{*}(\eta) \operatorname{sgn}\left(\eta_{\max }-|\eta|\right)$, where $k$ is a positive number with a lower boundary $k_{0}$. Here, $\operatorname{sgn}^{*}(\cdot)$ and sgn $(\cdot)$ are two symbolic functions defined as follows:

$$
\begin{aligned}
& \operatorname{sgn}^{*}(x)= \begin{cases}1, & x \geq 0, \\
-1, & x<0,\end{cases} \\
& \operatorname{sgn}(x)= \begin{cases}1, & x>0, \\
0, & x=0, \\
-1, & x<0\end{cases}
\end{aligned}
$$

As a consequence, the acceleration $a$ can be obtained as

$$
\begin{aligned}
a= & \frac{V^{2}(\cos \eta-1)(2 N-1)}{R \sin \eta \cos \eta}+\frac{V^{2} \sin \eta}{2 R} \\
& +V \dot{\gamma}-k s \cdot \operatorname{sgn}^{*}(\eta) \operatorname{sgn}\left(\eta_{\max }-|\eta|\right) .
\end{aligned}
$$

Therefore, followed by Equation (17), the derivative of $R, \eta, s$ alongside the close-loop system can be redefined as follows:

$$
\begin{aligned}
\dot{R}= & -V \cos \eta \\
\dot{\eta}= & \dot{\theta}-\dot{\gamma}=\frac{a}{V}-\dot{\gamma}=\frac{V(\cos \eta-1)(2 N-1)}{R \sin \eta \cos \eta}+\frac{V \sin \eta}{2 R} \\
& -\frac{k s \cdot \operatorname{sgn}^{*}(\eta) \operatorname{sgn}\left(\eta_{\max }-|\eta|\right)}{V}, \\
\dot{s}=- & \frac{R \sin \eta \cos \eta}{V^{2}(2 N-1)} k s \cdot \operatorname{sgn}^{*}(\eta) \operatorname{sgn}\left(\eta_{\max }-|\eta|\right) .
\end{aligned}
$$

\subsection{Stability Analysis of Acceleration Instruction}

Theorem 1. Supposing that the initial lead angle of missile $\eta_{0} \in(0, \pi / 2]$ satisfies $\left|\eta_{0}\right| \leq \eta_{\max }$, the antiship missile will attack the target accurately at a desired impact time $t_{d}$ under the drive of acceleration instruction $a$ in Equation (17) with the constraint of the FOV by choosing appropriate parameters $N, k$.

Proof. In order to prove Theorem 1, it is firstly to prove that in the whole terminal guidance process, the FOV will be constrained with the upper boundary $\eta_{\max }$.

Choosing a Lyapunov function $V_{1}$,

$$
V_{1}=\frac{1}{2} \eta^{2}
$$

Substituting Equation (19) into the derivation of $V_{1}$, one can gain

$$
\begin{gathered}
\dot{V}_{1}=\eta \dot{\eta}=\frac{V(\cos \eta-1)(2 N-1)}{R \sin \eta \cos \eta}+\frac{V \sin \eta}{2 R} \\
-\frac{k s|\eta| \operatorname{sgn}\left(\eta_{\max }-|\eta|\right)}{V} .
\end{gathered}
$$




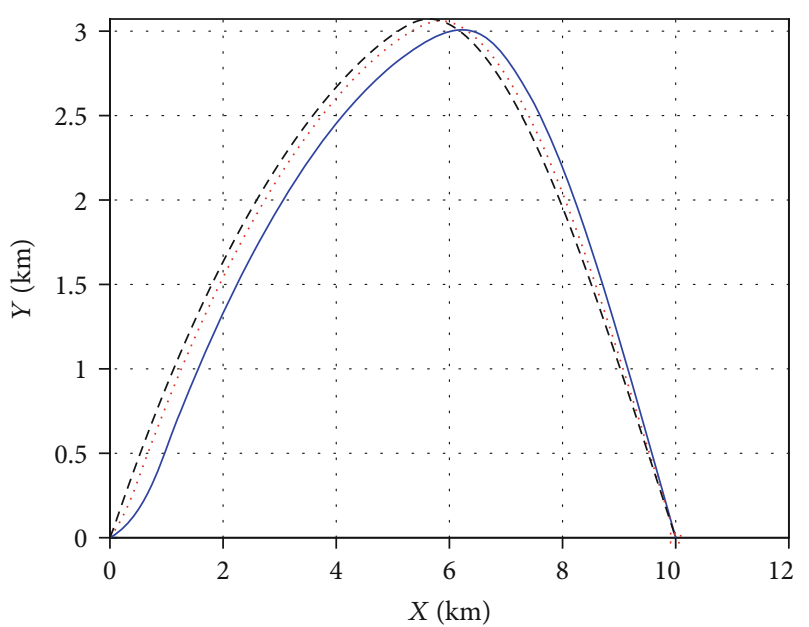

$$
\begin{aligned}
\eta & =10^{\circ} \\
\eta & =25^{\circ} \\
--\eta & =40^{\circ}
\end{aligned}
$$

(a) Trajectory of missile

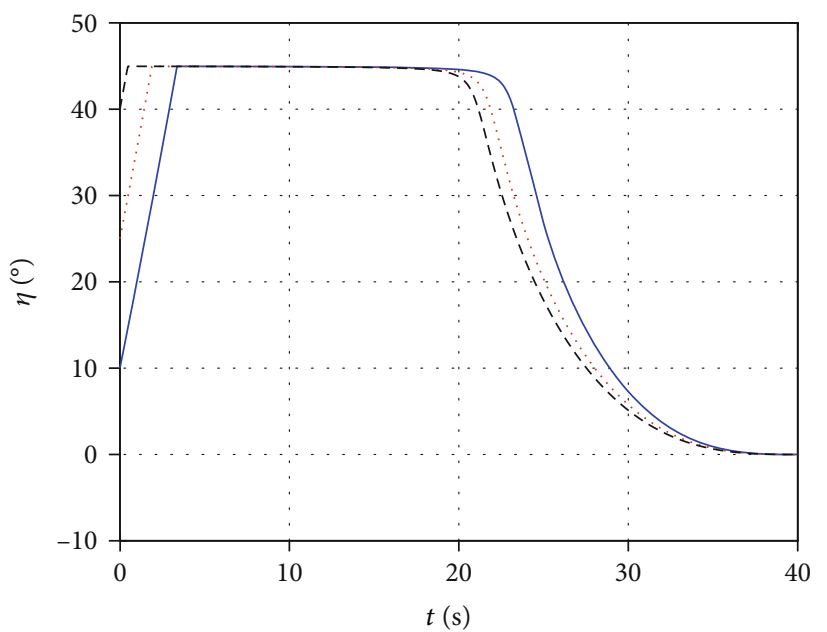

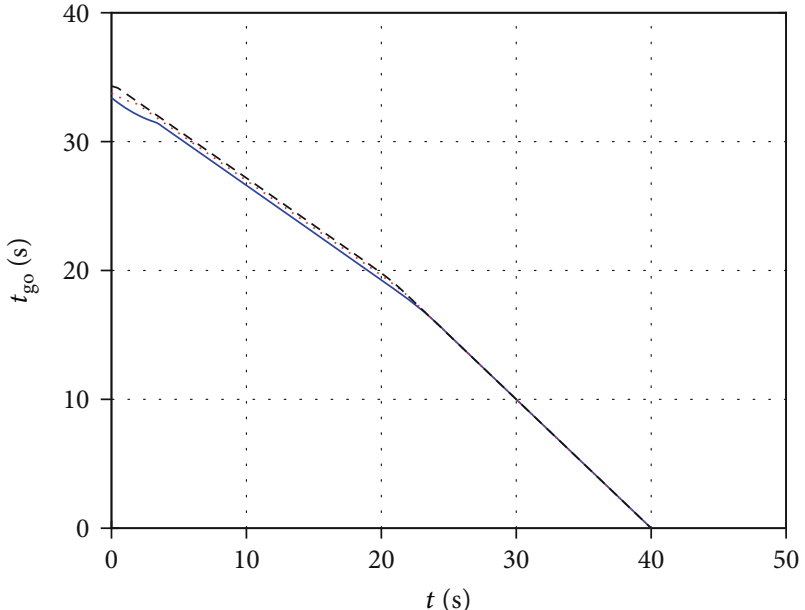

$$
\begin{aligned}
\eta & =10^{\circ} \\
\ldots \ldots \eta & =25^{\circ} \\
--\eta & =40^{\circ}
\end{aligned}
$$

(b) Remaining flying time

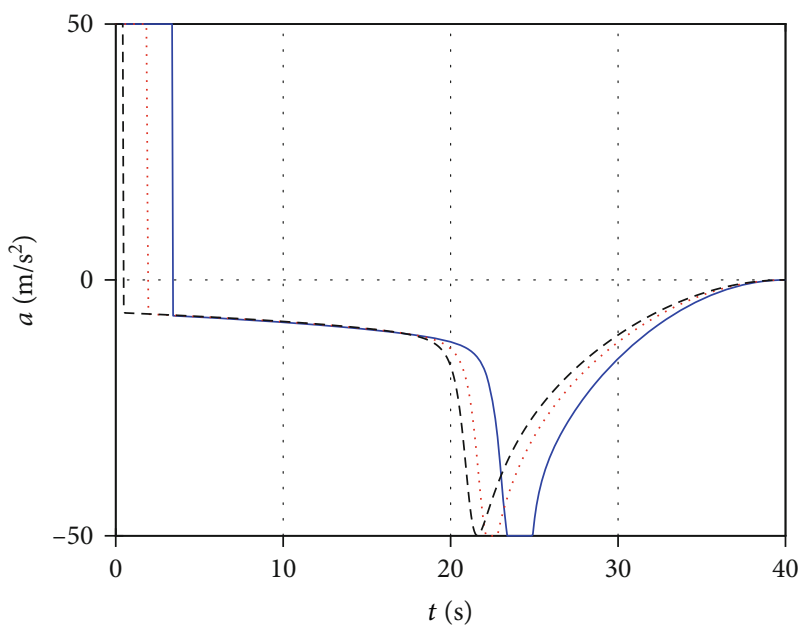

$$
\begin{aligned}
\eta & =10^{\circ} \\
\cdots \ldots . & =25^{\circ} \\
\eta & =40^{\circ}
\end{aligned}
$$

(d) Acceleration instruction

(c) Lead angle

Figure 2: Continued. 


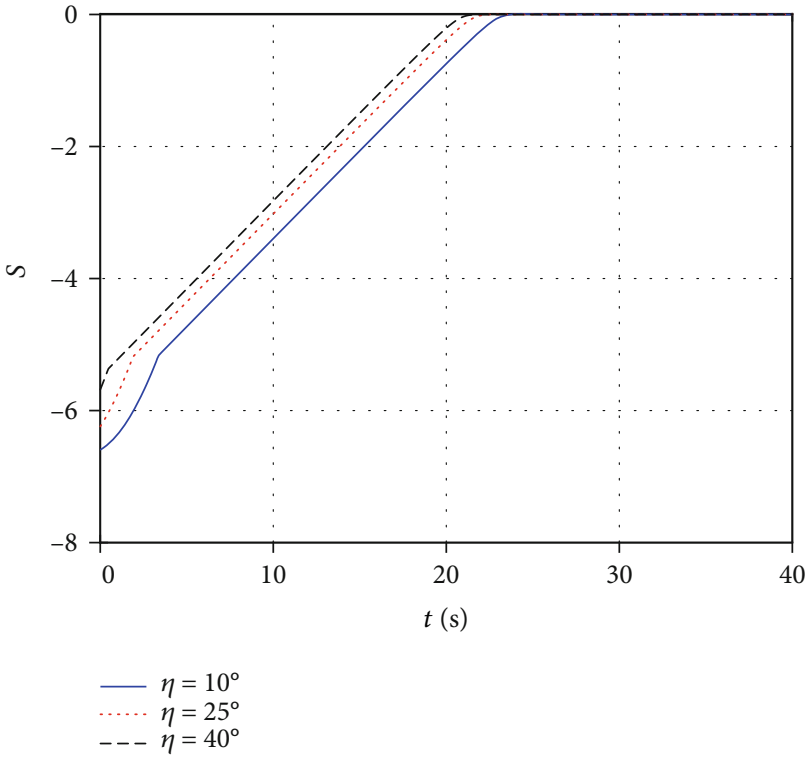

(e) Sliding mode surface

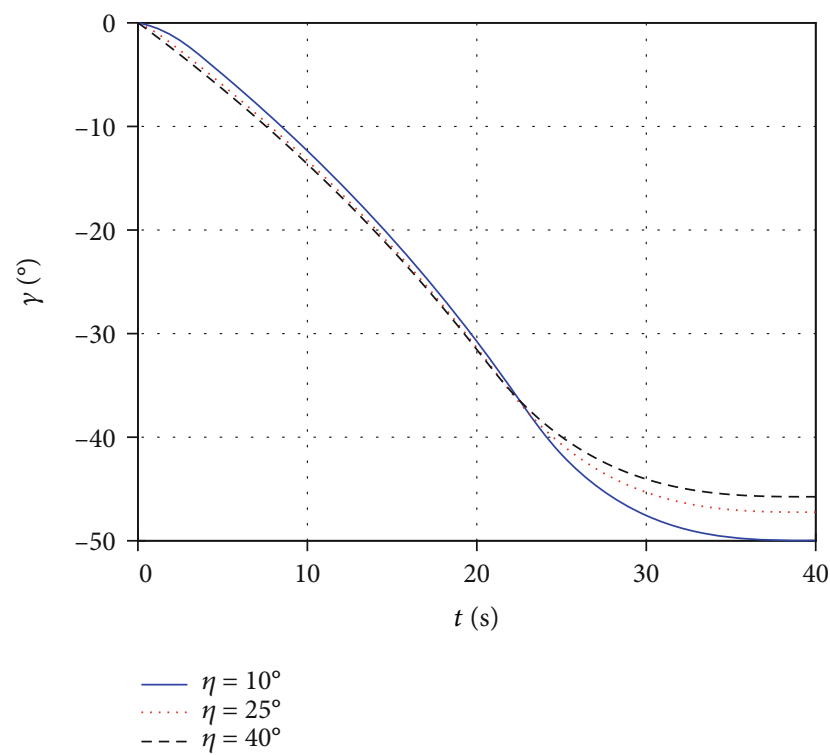

(f) LOS angle

FIGURE 2: Simulation results of case 1.

Supposing that FOV reaches the upper boundary at instant $t_{s}, t_{s} \in\left[0, t_{d}\right]$, that is $\left|\eta\left(t_{s}\right)\right| \leq \eta_{\max }$, Equation (22) is converted into

$$
\begin{aligned}
\dot{V}_{1} & =\frac{V\left(\cos \eta_{\max }-1\right)(2 N-1)}{R \sin \eta_{\max } \cos \eta_{\max }}+\frac{V \sin \eta_{\max }}{2 R} \\
& =\underbrace{-\frac{V(2 N-1)}{R \sin \eta_{\max } \cos \eta_{\max }}}_{V_{a}} \underbrace{\left[1-\cos \eta_{\max }\left(1+\frac{\sin ^{2} \eta_{\max }}{2(2 N-1)}\right)\right]}_{V_{b}} \\
& =V_{a} V_{b} .
\end{aligned}
$$

Thus, the positive and negative value of $\dot{V}_{1}$ depends on the result of the multiplication of $V_{a} V_{b}$. During the terminal guidance process, restricted with the influence of actual combat scenarios, the relative range $R>0$ holds definitely. Since $\eta_{0} \in(0, \pi / 2], V_{a}<0$. And the positive and negative value of $V_{b}$ is discussed as follows:

Defining a function $g(x), x \in(0, \pi / 2]$,

$$
g(x)=1-\cos x\left(1+\frac{\sin ^{2} x}{2(2 N-1)}\right)
$$

The derivation of $g(x)$ can be expressed as

$$
\begin{aligned}
\dot{g}(x) & =\sin x-\frac{2 \cos ^{2} x \sin x-\sin ^{3} x}{2(2 N-1)} \\
& =\sin x\left[1-\frac{\cos ^{2} 2 x+\cos ^{2} x}{2(2 N-1)}\right]>0,
\end{aligned}
$$

which demonstrates that $g(x)$ is a monotonically increasing function with $x \in(0, \pi / 2]$.

As a result, $g(x)_{\min }=\lim _{x \rightarrow 0^{+}} g(x)=0$, which implies that $V_{b}>0$ is established all the time. Therefore, $\dot{V}_{1}<0$ holds.

Consequently, in the terminal guidance process, $\left|\eta\left(t_{s}\right)\right|$ $\leq \eta_{\max }$ holds at arbitrary $t_{s}$, which means that the constraint of FOV can be guaranteed. Moreover, only if $\eta\left(t_{s}\right)$ is equal to $\eta_{\max }$, the FOV will reach the upper boundary. When $s \geq 0$, it can be obtained that $|\eta|$ monotonously decreases followed by Equation (22), which implies that $\left|\eta\left(t_{s}\right)\right| \leq \eta_{\max }$ is always established. On the other hand, if $s<0$, the sign of Equation (22) is uncertain at this moment. However, Equation (22) is a continuous function made up of $\eta$ with $\dot{V}_{1}\left(\eta_{\max }\right)<0$. Thus, there must exist a constant $\delta \in \mathbb{R}^{+}$which satisfies $\dot{V}_{1}\left(\eta_{\max }-\delta\right)=0$ so that $\left|\eta\left(t_{s}\right)\right| \leq \eta_{\max }-\delta$ holds throughout the terminal guidance. As a result, when $\eta\left(t_{s}\right)=\eta_{\max }, \dot{V}_{1}$ will decrease to be $\dot{V}_{1}\left(\eta_{\max }-\delta\right)=0$ gradually and then varies according to the sliding mode surface $s$. Therefore, the constraint of FOV can be guaranteed in the whole terminal process.

Then, it is essential to address that under the action of the designed acceleration instruction $a$ in Equation (17), the sliding mode surface will converge to be zero.

Selecting a Lyapunov function $V_{2}$,

$$
V_{2}=\frac{1}{2} s^{2}
$$

Taking the derivative of $V_{2}$ along the close-sloop system and combining with Equation (20) together, one can have 

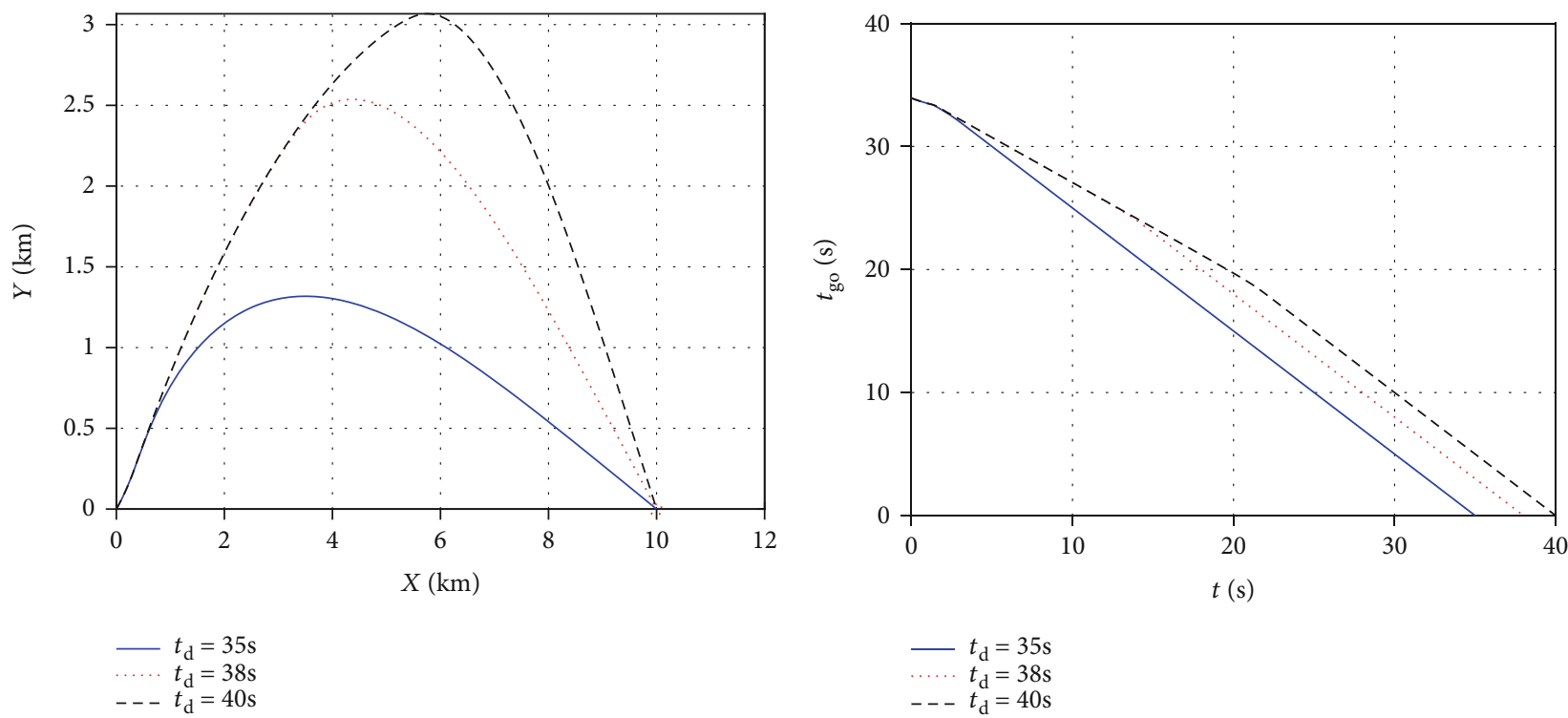

(a) Trajectory of missile
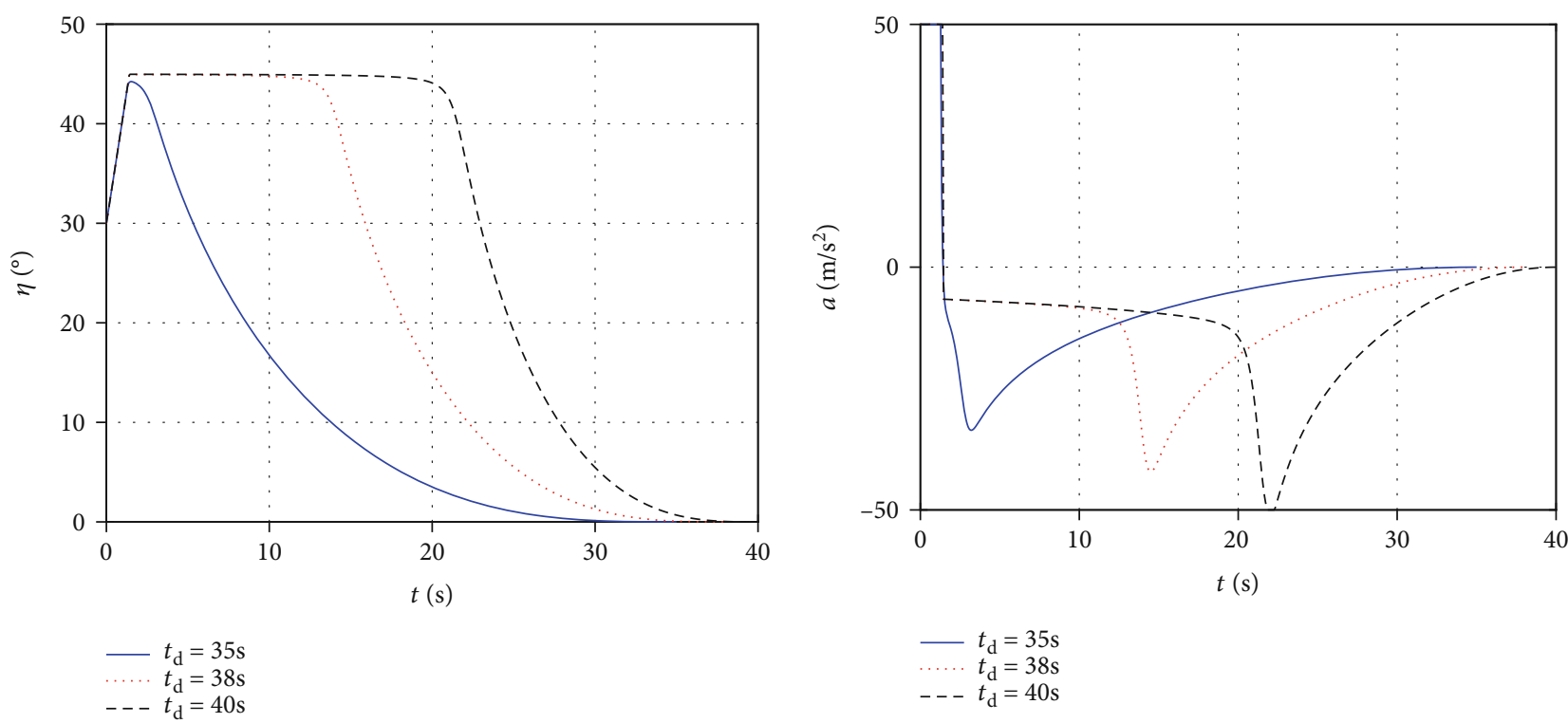

(c) Lead angle

(d) Acceleration instruction

Figure 3: Continued. 


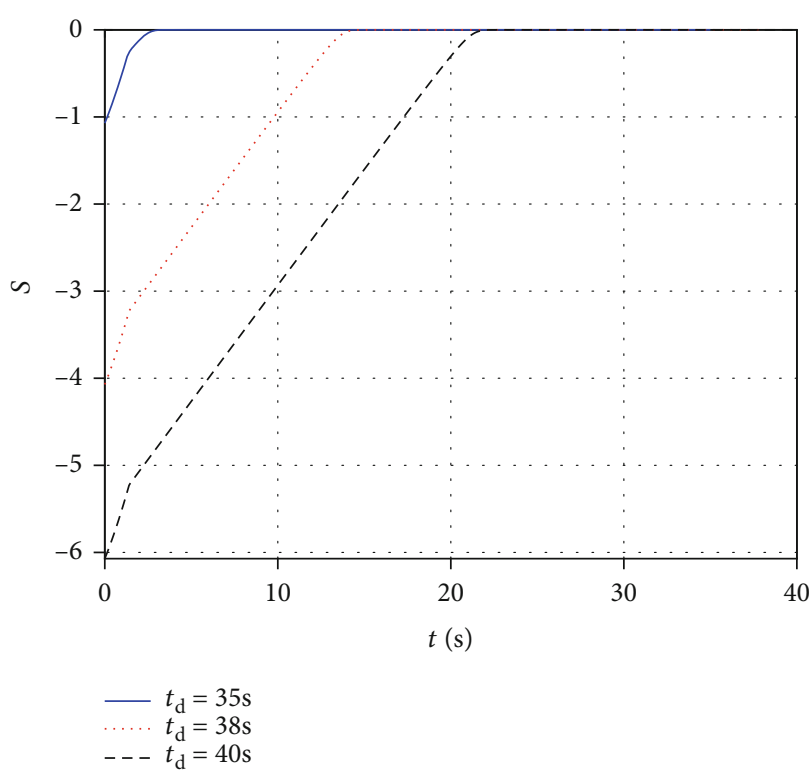

(e) Sliding mode surface

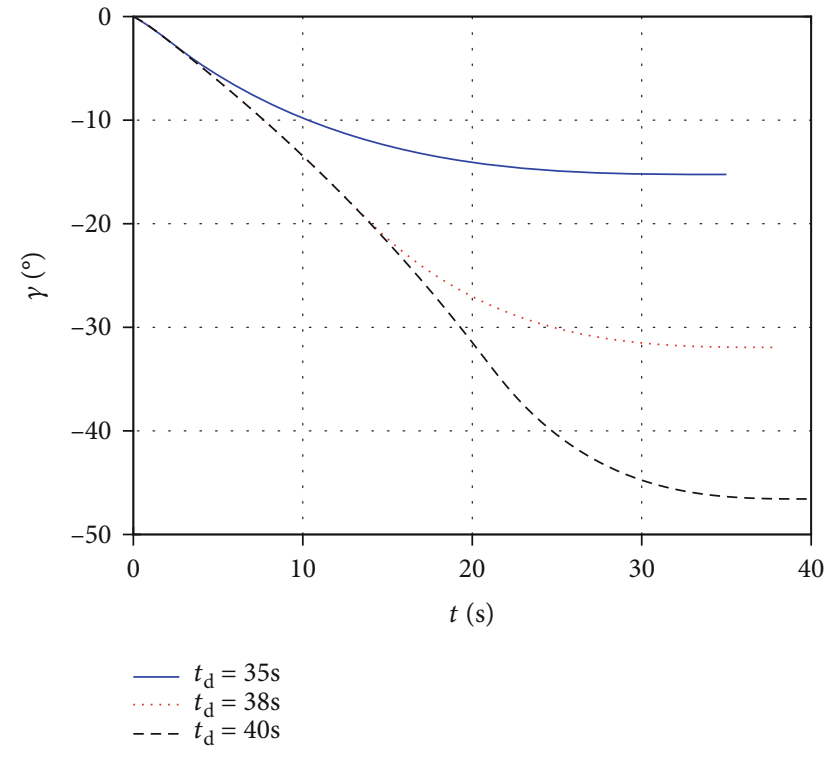

(f) LOS angle

Figure 3: Simulation results of case 2.

$$
\begin{aligned}
\dot{V}_{2} & =s \dot{s}=-\frac{R \sin \eta \cos \eta}{V^{2}(2 N-1)} k s^{2} \cdot \operatorname{sgn}^{*}(\eta) \operatorname{sgn}\left(\eta_{\max }-|\eta|\right) \\
& =-\frac{R|\sin \eta| \cos \eta}{V^{2}(2 N-1)} k s^{2} \cdot \operatorname{sgn}\left(\eta_{\max }-|\eta|\right) \leq 0
\end{aligned}
$$

where $|\sin \eta|=\sin \eta \cdot \operatorname{sgn}^{*}(\eta)$.

Thus, the acceleration ensures to make sliding mode surface converge to be zero in the stability of Lyapunov sense. Therefore, Theorem 1 is proved.

Furthermore, as is shown in Equations (17) and (19), there is a component element composed of lead angle $\eta$ correspondingly, it is necessary to discuss the singularity phenomenon when $\eta\left(t_{s}\right) \longrightarrow 0, t_{s} \in\left[0, t_{d}\right]$.

For the first part of Equation (17), by applying the Law of Robida, one can yield

$$
\begin{aligned}
\lim _{\eta \rightarrow 0} \frac{V^{2}(\cos \eta-1)(2 N-1)}{R \sin \eta \cos \eta} \\
=\frac{V^{2}(2 N-1)}{R} \lim _{\eta \rightarrow 0} \frac{(\cos \eta-1)}{\sin \eta \cos \eta} \\
=\frac{V^{2}(2 N-1)}{R} \lim _{\eta \rightarrow 0} \frac{-\sin \eta}{\cos ^{2} \eta-\sin ^{2} \eta}=0 .
\end{aligned}
$$

Similarly, with respect to the first part of Equation (19), one can obtain

$$
\begin{aligned}
\lim _{\eta \longrightarrow 0} \frac{V(\cos \eta-1)(2 N-1)}{R \sin \eta \cos \eta} \\
=\frac{V(2 N-1)}{R} \lim _{\eta \longrightarrow 0} \frac{(\cos \eta-1)}{\sin \eta \cos \eta} \\
=\frac{V(2 N-1)}{R} \lim _{\eta \longrightarrow 0} \frac{-\sin \eta}{\cos ^{2} \eta-\sin ^{2} \eta}=0 .
\end{aligned}
$$

It can be seen from Equations (28) and (29) that when sliding mode surface approaches to be zero, both acceleration instruction $a$ and the rate of lead angle $\dot{\eta}$ tend to be zero, respectively, which demonstrates that there is no singularity phenomenon in them, implying that there will be no sudden jumps in acceleration instruction.

In addition, according to the stability analysis of acceleration instruction, it is of great of importance to select parameter $k$ in Equation (17) to ensure that $s$ converges to be zero in preference to $\eta$. Based on Equation (19), the boundary of $k$ can be given as follows:

$$
k>\frac{1}{\operatorname{sgn}^{*}\left(\eta_{0}\right) s\left(t_{0}\right)}\left[\frac{\left(1-\cos \eta_{0}\right)}{R_{0} \eta_{0}} V(2 N-1)-\frac{V \eta_{0} \cos \eta_{0}}{2 R_{0}}\right],
$$

where subscript " 0 " indicates the initial moment of terminal guidance.

\section{Simulation Results}

In order to perform the effectiveness of the proposed guidance law with desired impact and FOV constrained. We conduct experimental verification from different aspects including four different simulation cases. In all simulations, the initial coordinates of the missile and target are located at $(0 \mathrm{~km}, 0 \mathrm{~km})$ and $(10 \mathrm{~km}, 0 \mathrm{~km})$ correspondingly. The speed of the missile is $300 \mathrm{~m} / \mathrm{s}$. The corresponding guidance parameters of acceleration instruction proposed in Equation (17) are selected with $k=280, N=4$. The limit of FOV and acceleration instruction are $\eta_{\max }=45^{\circ}$ and $a_{\max }=50 \mathrm{~m} / \mathrm{s}^{2}$ separately. Besides, on the premise of ensuring the stability of the guidance system, in order to minimize signal chatter caused by the sign function existing in Equation (17), a 


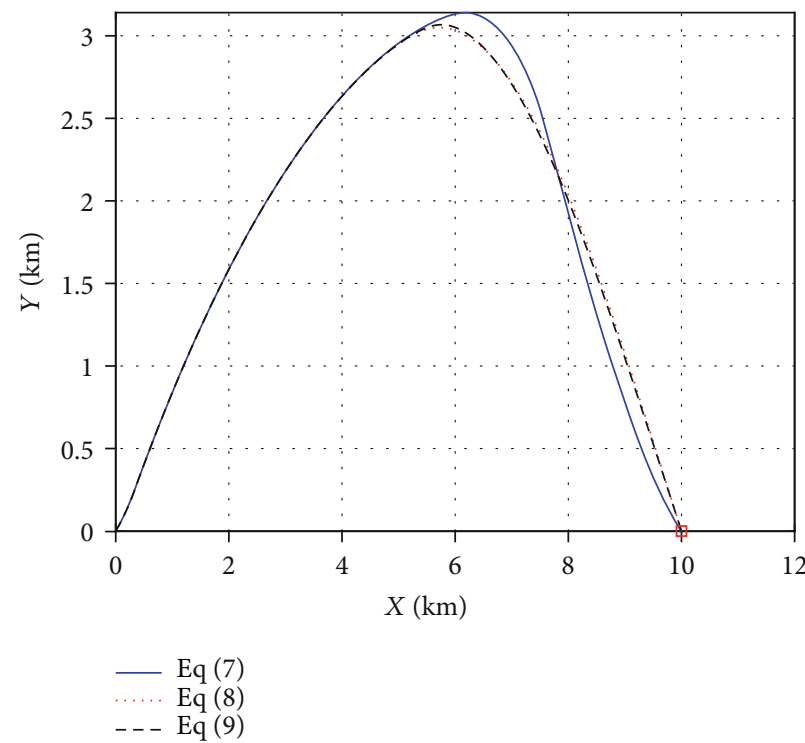

(a) Trajectory of missile

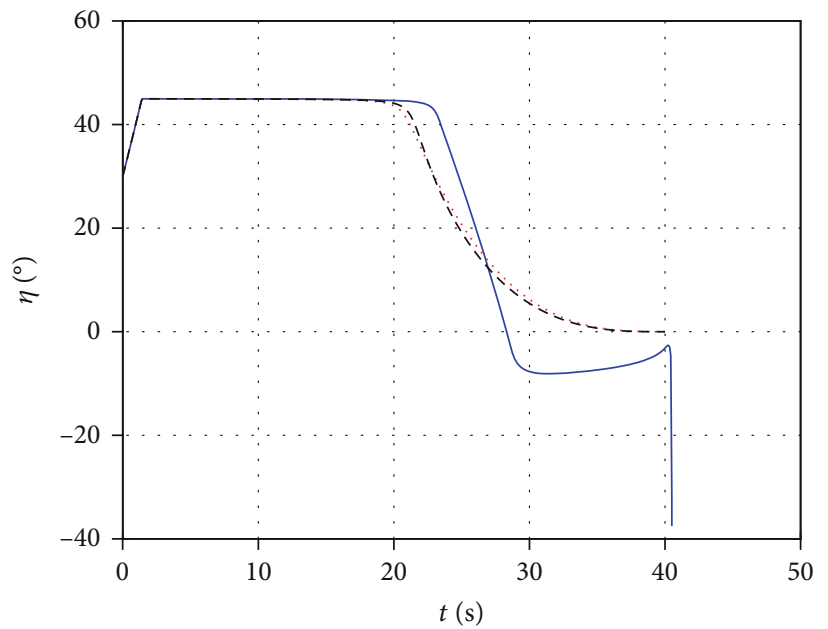

$-\mathrm{Eq}(7)$
$\ldots . . \mathrm{Eq}(8)$
$\ldots-$.

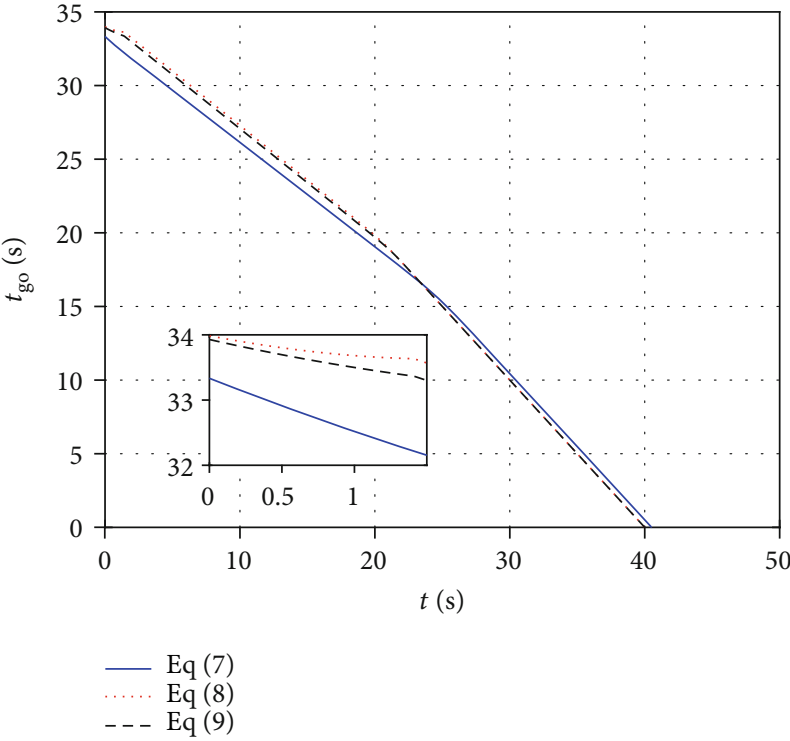

(b) Remaining flying time

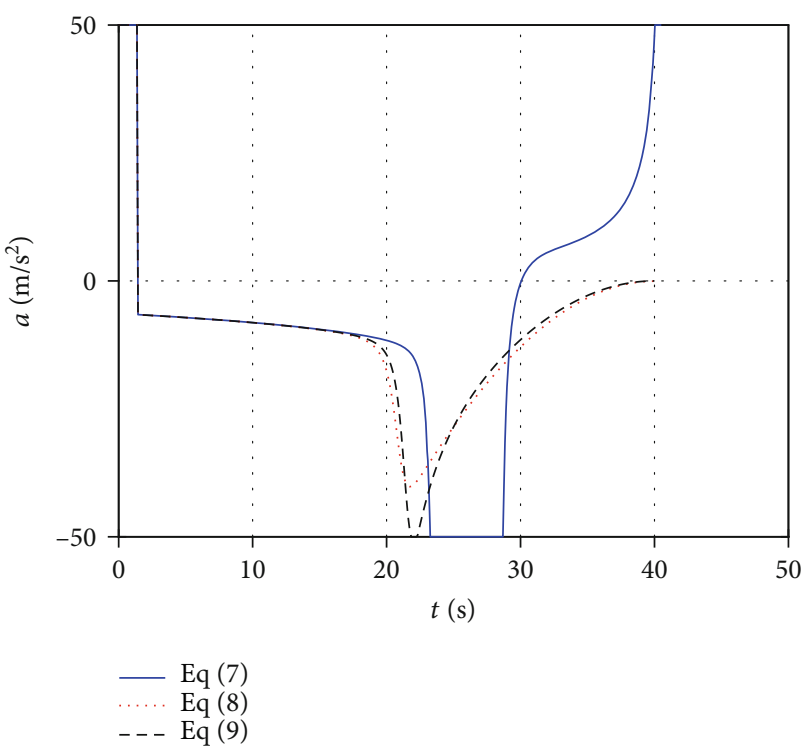

(d) Acceleration instruction

Figure 4: Continued. 


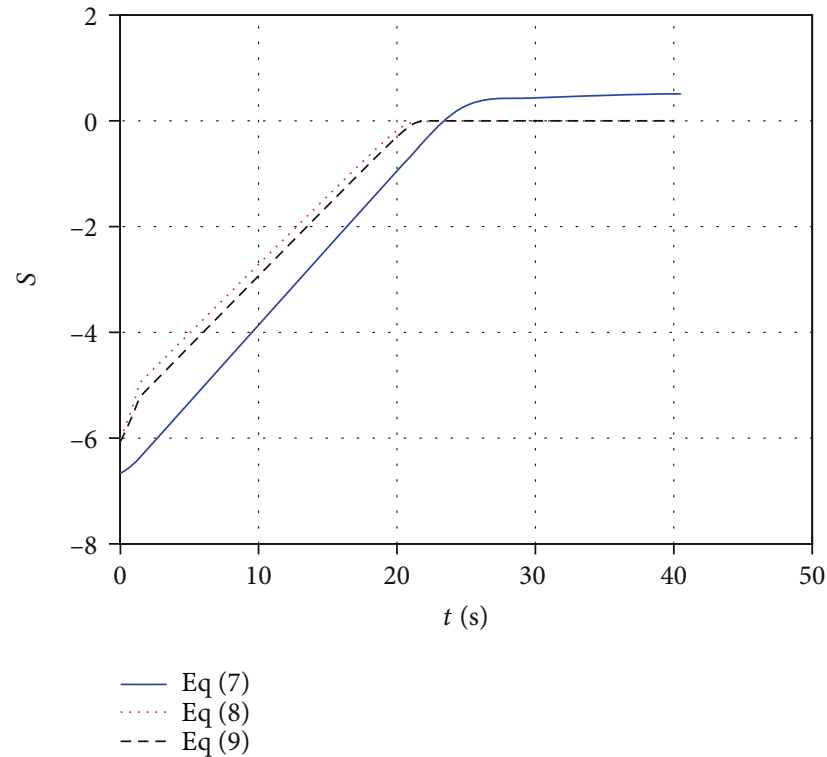

(e) Sliding mode surface

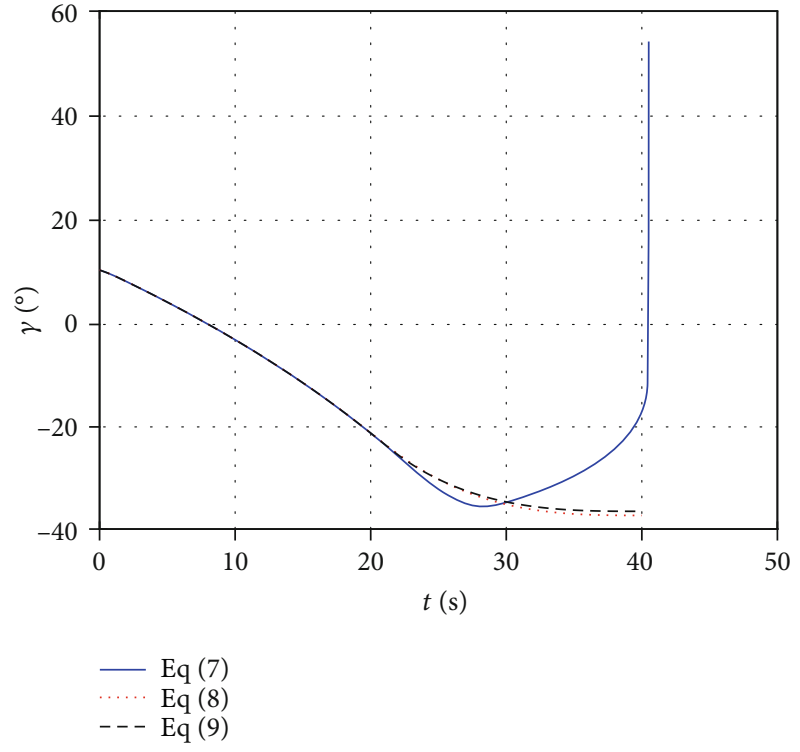

(f) LOS angle

FIGURE 4: Simulation results of case 3.

continues function is adopted to replace the sign function, which is expressed as

$$
\operatorname{sgmf}(x)=2\left(\frac{1}{1+\exp ^{-\tau x}}-\frac{1}{2}\right), \quad a>0,
$$

where $\tau$ is chosen as 20 .

4.1. Attack with Fixed Impact Time and Different Initial Lead Angle. In this subsection, simulation is operated with $\eta_{0}=$ $10^{\circ}, 25^{\circ}, 40^{\circ}$ and fixed impact time $t_{d}=40 \mathrm{~s}$ to attack the target. The simulation results are illustrated in Figure 2. It can be seen from Figure 2(a) that the target can be successfully attacked with different initial lead angles. And the larger the lead angle, the more to the left of the highest point of the trajectory. Figure 2(b) shows that the missile will adjust the remaining flying time at the beginning of terminal guidance process in the presence of an initial heading error so that the missile moves towards the target all the time. As is shown in Figures 2(c)-2(e), the proposed acceleration instruction works effectively to guarantee the constraint of FOV. As the lead angle reaches the upper boundary, the acceleration instruction reduces rapidly to ensure the limitation of FOV. At the moment of sliding mode surface converging to be zero, the lead angle decreases to be zero monotonously. Afterwards, sliding mode surface stays on zero, which implies that the desired impact can be satisfied. In Figure 2(f), the impact angle varies with different initial lead angle, which implies that the effectiveness of damage to target can be improved by adjusting the initial lead angle.

4.2. Attack with Fixed Initial Lead Angle and Different Impact Time. In this subsection, simulation is operated with $t_{d}=35 \mathrm{~s}, 38 \mathrm{~s}, 40 \mathrm{~s}$ and fixed initial lead angle $\eta_{0}=30^{\circ}$ to
TABle 1: Parameters of cooperative attack.

\begin{tabular}{lccc}
\hline Number & Position $(\mathrm{km})$ & Initial lead angle $\left(^{\circ}\right)$ & Speed $(\mathrm{m} / \mathrm{s})$ \\
\hline M1 & $(0.0)$ & 30 & 300 \\
M2 & $(1.4)$ & 39 & 300 \\
M3 & $(0.3)$ & 27 & 300 \\
\hline
\end{tabular}

attack the target. The simulation results are presented in Figure 3. As is shown in Figure 3(a), impact time has much more influence on the curvature of the trajectory of missile than the initial lead angle. The larger the impact time, the greater the height of the trajectory that needs to be raised in order to satisfy the impact time constraint. In Figure 3(b), at the beginning of terminal guidance process, the change of remaining flying time varies small because of the same initial lead angle. Subsequently, it changes apparently due to different constraints of impact time. It can be seen from Figures 3(c)-3(e) that the proposed acceleration instruction can guarantee that the missile attacks the target with different desired impact and the FOV is limited. The larger the impact time constraint, the greater the magnitude of the acceleration instruction change so that it takes longer time for the sliding mode surface to converge to be zero. In Figure 3(f), impact time has much more influence on the magnitude of LOS angle than the initial lead angle, which demonstrates that by setting an appropriate impact time can satisfy the constraint of FOV and improve the effectiveness of damage to target.

4.3. Compared with Other Methods of Estimating $t_{g o}$. In this subsection, in order compare the effectiveness of methods of estimating $t_{\text {go }}$ mentioned in Equations (7)-(9), simulation is operated with $\eta_{0}=30^{\circ}$ and fixed impact time $t_{d}=40 \mathrm{~s}$ to 

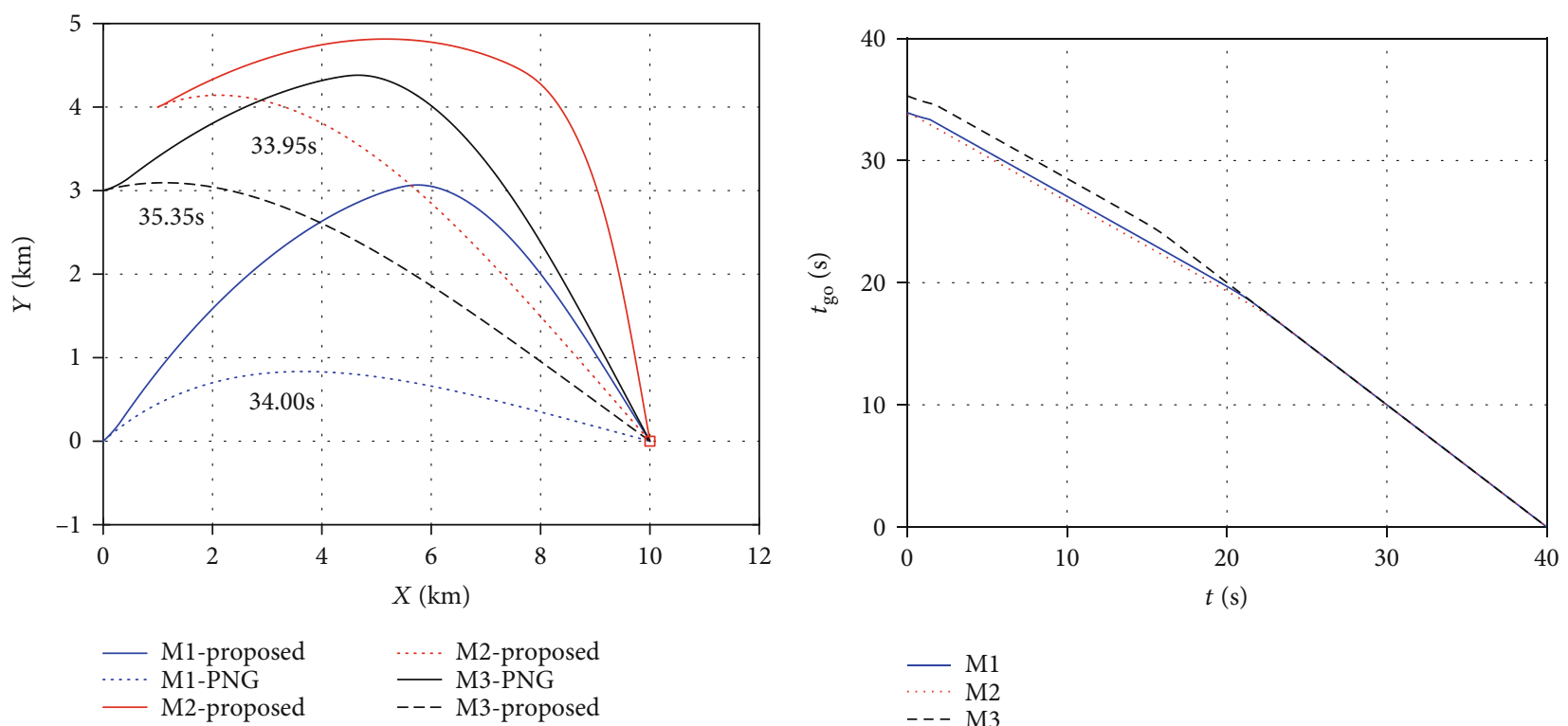

(a) Trajectory of missile
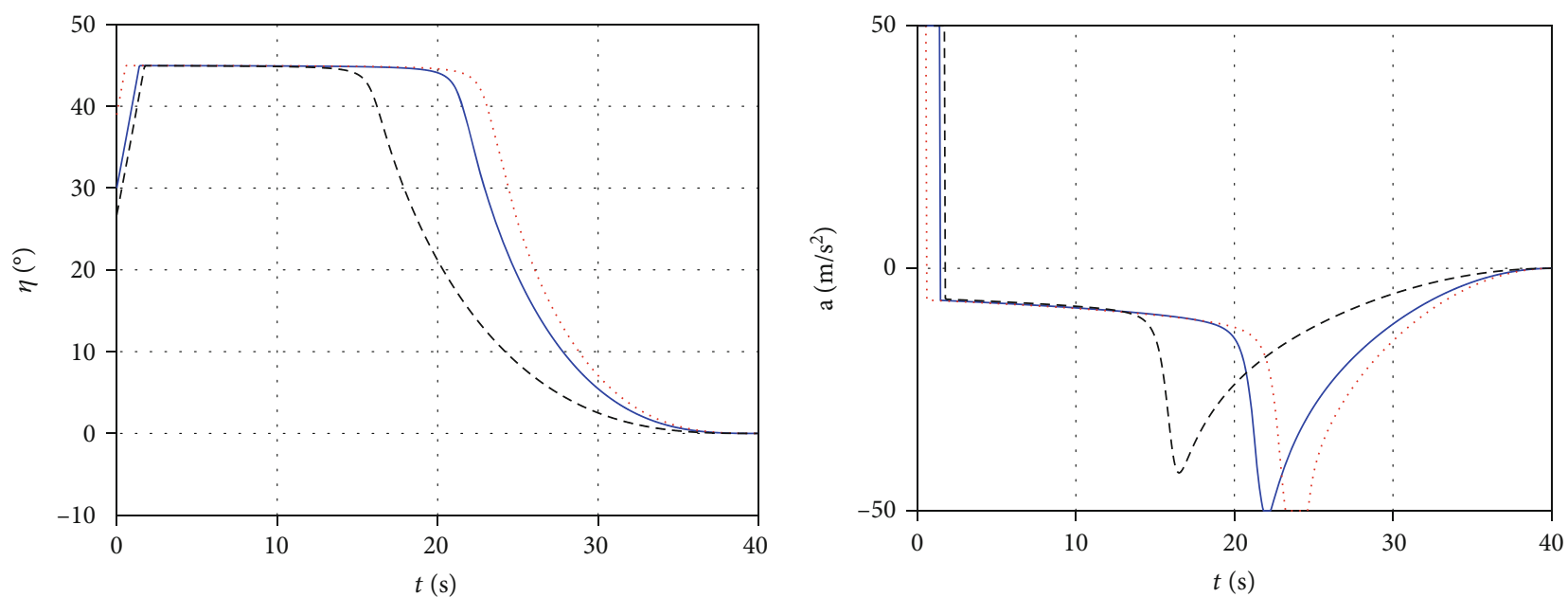

$\begin{array}{rr}- & \mathrm{M} 1 \\ \ldots . . . & \mathrm{M} 2 \\ \ldots & \mathrm{M} 3\end{array}$

$-\mathrm{M} 1$

…. M2

- - M3

(c) Lead angle

(d) Acceleration instruction

FIgUre 5: Continued. 


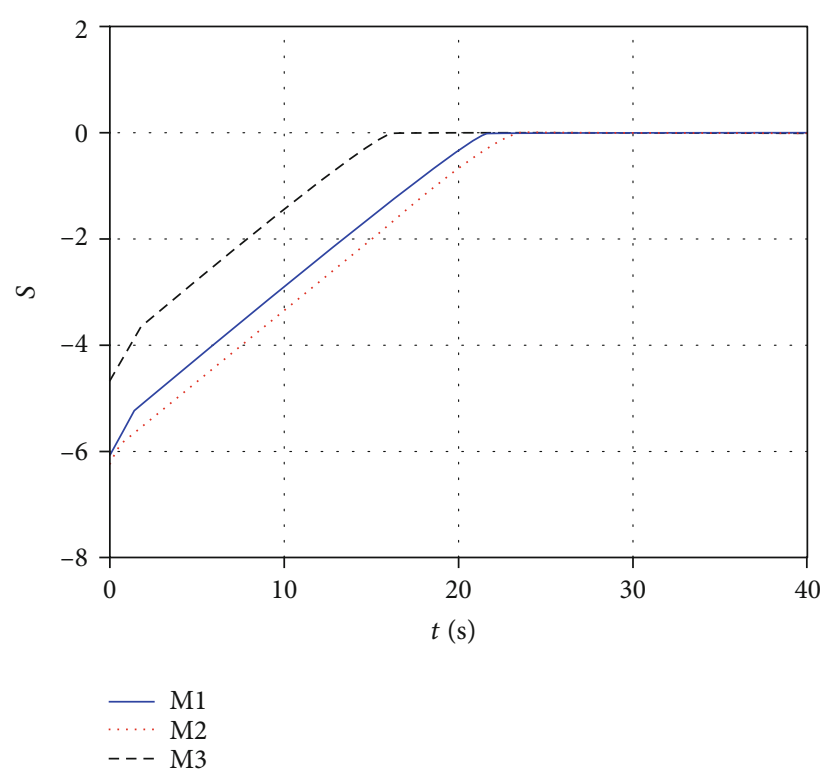

(e) Sliding mode surface

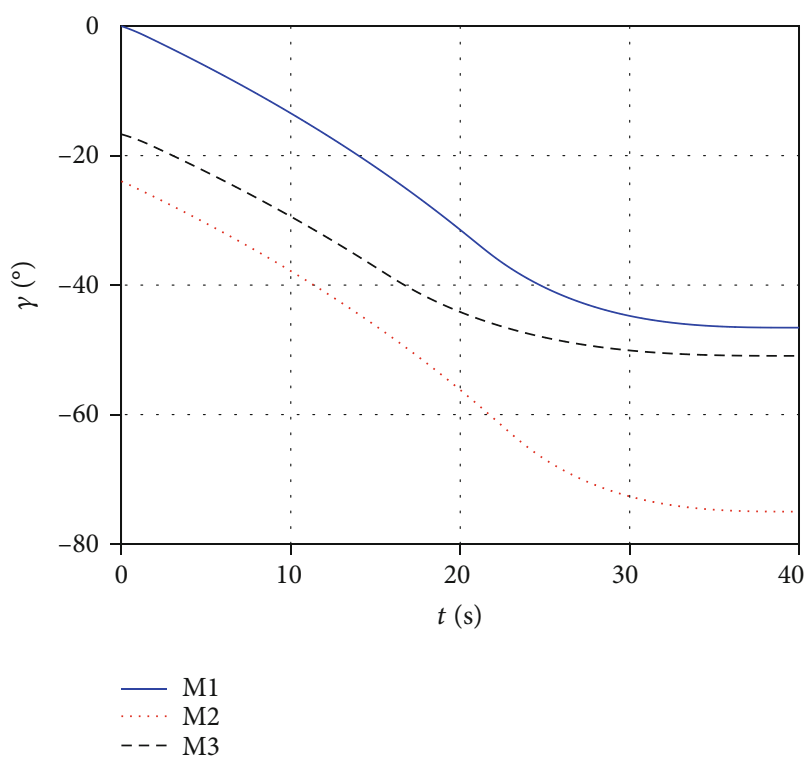

(f) LOS angle

FiguRe 5: Simulation results of case 4.

attack the target. The simulation results are illustrated in Figure 4. It can be seen from Figure 4(a) that the missile can attack the target based on different methods of estimating $t_{\mathrm{go}}$. In terms of attack accuracy, the corresponding miss distances are $1.93 \mathrm{~m}, 0.46 \mathrm{~m}$, and $0.30 \mathrm{~m}$, which demonstrates the superiority of Equation (9). As is shown in Figure 4(b), Equation (9) shows its advantage in the calculation accuracy of $t_{\mathrm{go}}$. For Equation (9), it is established on the basis of the missile's own motion characteristics and there is no need to apply the small-angle assumption, which results in promoting the actual application value. In Figure 4(c) and 4(f), the lead angle and the value of lead angle and LOS angle in Equation (8) and Equation (9) vary approximately the same to some extent. However, for Equation (7), it gradually changes at about $20 \mathrm{~s}$, which may bring uncertainty to the safe flight of the missile. It can be seen from Figure 4(d) that acceleration instruction in Equations (8) and (9) varies smoothly and reasonably during the whole process of attack. For Equation (7), the acceleration instruction appears to be saturated, which results in imposing a great burden to the missile. In Figure 4(e), the values of impact time errors are $-0.51 \mathrm{~s}, 0.10 \mathrm{~s}$, and $0.01 \mathrm{~s}$, which demonstrates that the remaining flying time estimated by Equation (9) is much more effective.

4.4. Multiple Missile Attack Cooperatively. In this subsection, simulation is conducted with multiple missiles to attack a stationary target cooperatively at a fixed impact time $t_{d}=$ $40 \mathrm{~s}$. The initial simulation conditions of missiles are shown in Table 1, and the simulation results are plotted in Figure 5. As is shown in Figure 5(a), in order to further demonstrate the superiority of the proposed acceleration instruction, simulation is operated compared with the PNG algorithm. In comparison to Equation (17), the impact times of missiles which are driven with the PNG algorithm are $34.00 \mathrm{~s}$,
$33.95 \mathrm{~s}$, and $35.35 \mathrm{~s}$, respectively, which is quite different from the desired time. On the other hand, by setting fixed impact, missiles can cooperate together to attack the target simultaneously, increasing attack efficiency on the target. It can be seen from Figure 5(b) that because of the distinction of initial position and lead angle among three missiles, there are some differences in the remaining flying time. Then, they gradually converge to be the same, ensuring a cooperative attack at the desired time. Figures 5(c)-5(e) demonstrate that the proposed acceleration instruction has good application value in the cooperative attack of target. The acceleration instruction varies reasonably, which ensures that the sliding mode surface of each missile converges to be zero smoothly, providing a basic guarantee for cooperative attack. In Figure 5(f), by setting various initial conditions of missiles, there are large LOS angles of three missiles at attack moment, which not only provides the accuracy of damage to target but also improves the damage effect on the target.

4.5. Expansion to a Target with Constant Speed. Most of the previous research mainly focused on stationary targets, and the corresponding experiments are not conducted on a target with a constant speed. Inspired by the concept of PIP, the target moving with a constant speed can be treated as a virtual stationary target with respect to the missile [1]. The position of target by using PIP theory can be expressed as follows:

$$
\begin{gathered}
X_{T_{P}}=X_{T}+V_{T} \cos \theta_{T} t_{\mathrm{go}}, \\
Y_{T_{P}}=Y_{T}+V_{T} \sin \theta_{T} t_{\mathrm{go}},
\end{gathered}
$$

where $\left(X_{T_{P}}, Y_{T_{P}}\right)$ is the PIP, $\left(X_{T}, Y_{T}\right)$ is for the instantaneous position of target, and $\theta_{T}$ is the ballistic inclination angle of target. 

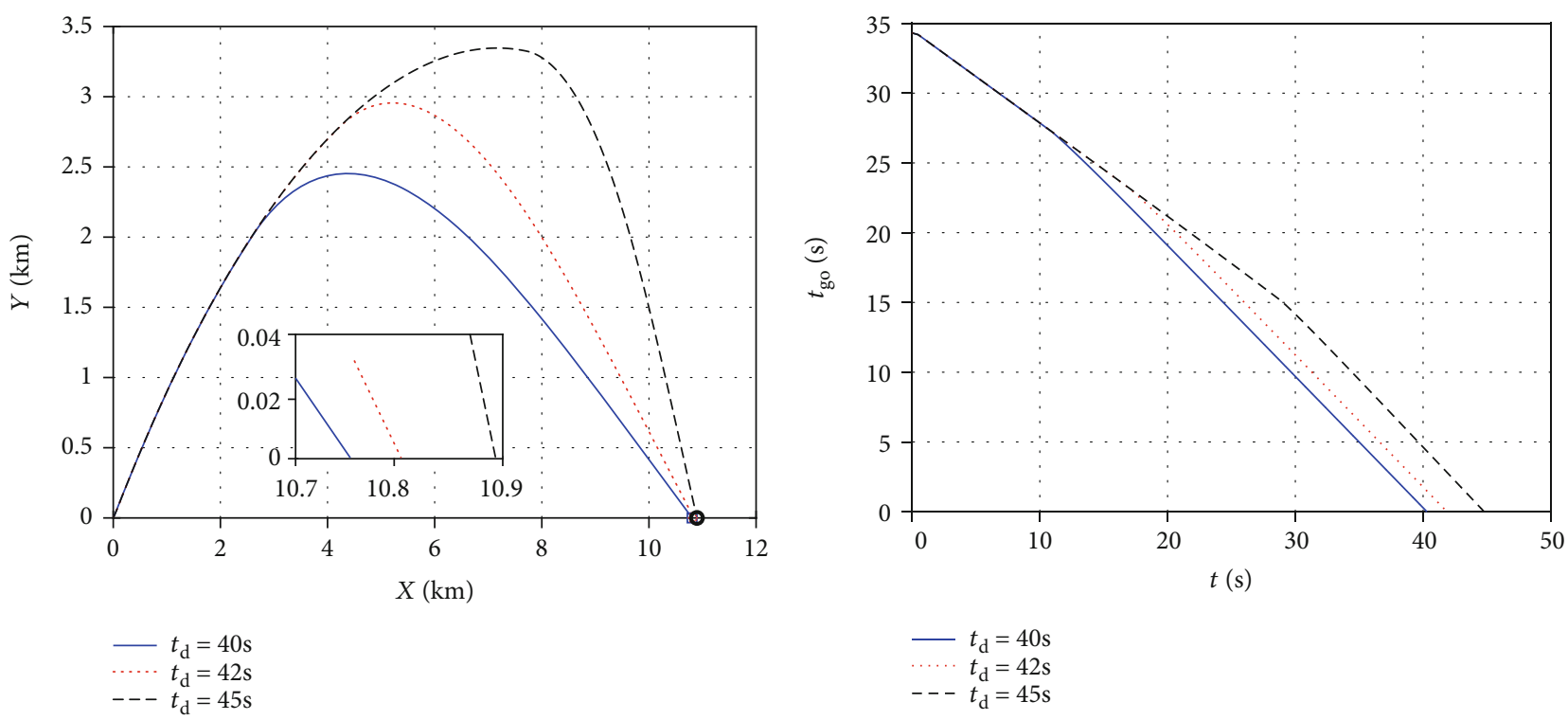

(a) Trajectory of missile

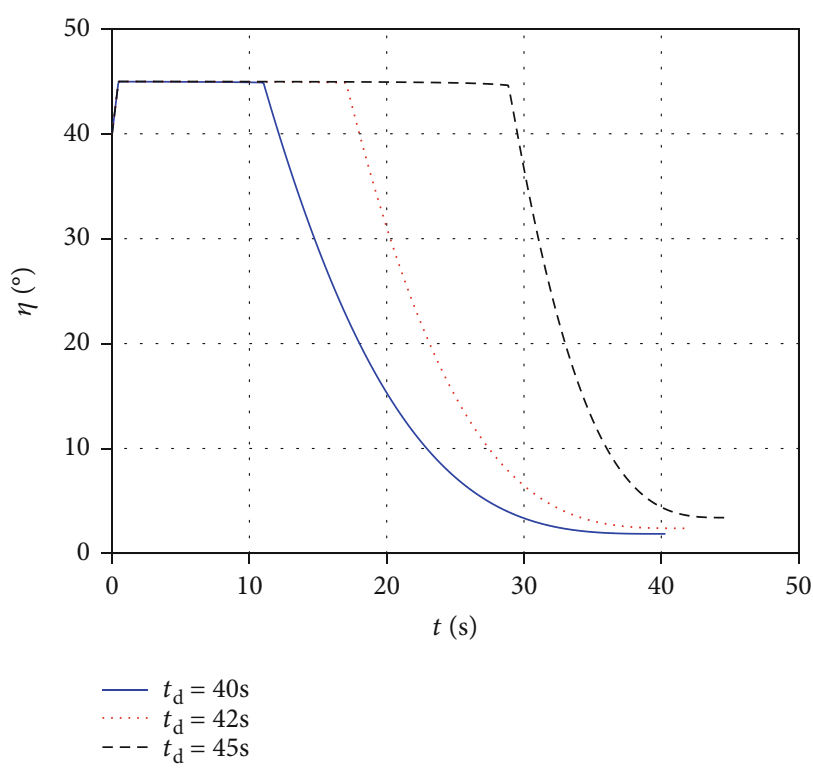

(b) Remaining flying time

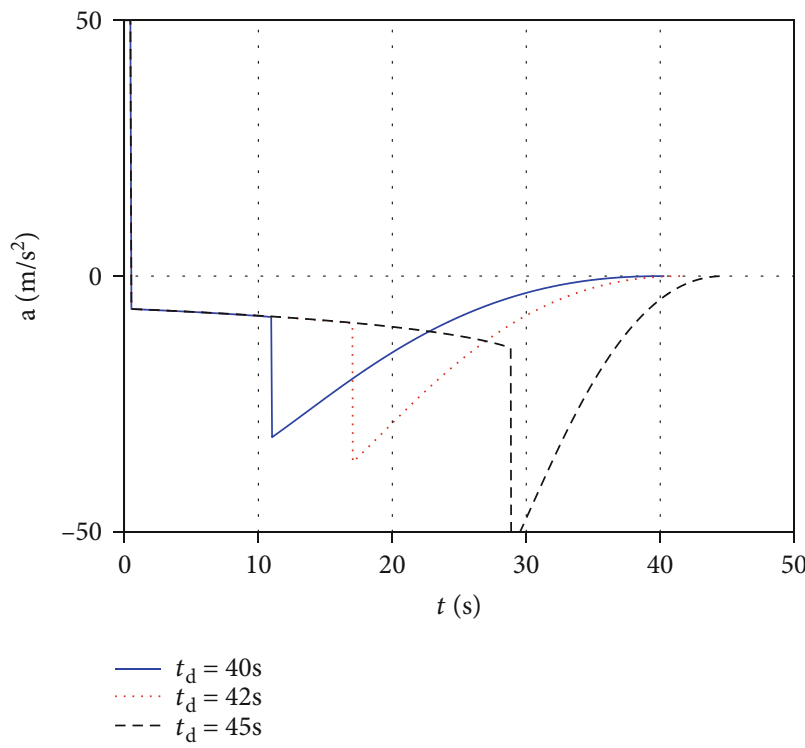

(c) Lead angle

(d) Acceleration instruction

Figure 6: Continued. 


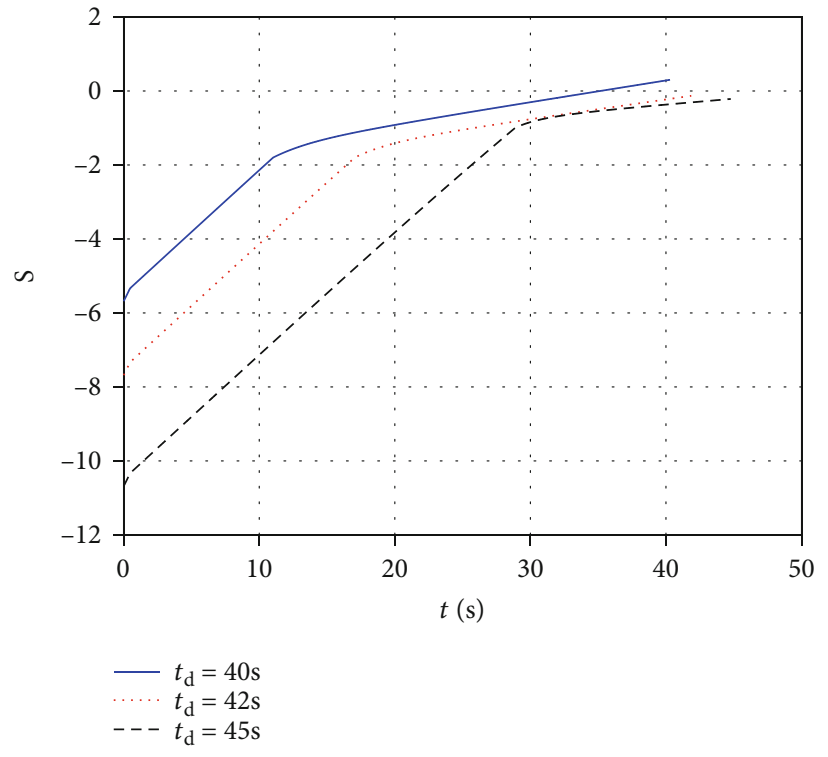

(e) Sliding mode surface

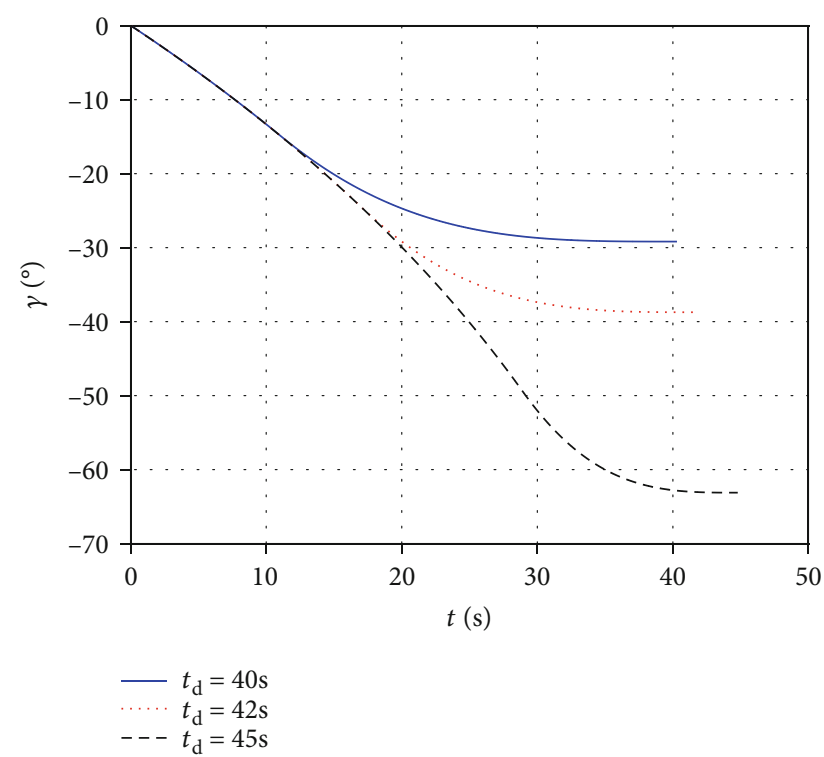

(f) LOS angle

Figure 6: Simulation results of case 5.

In this subsection, simulation is conducted with $t_{d}=$ $40 \mathrm{~s}, 42 \mathrm{~s}, 45 \mathrm{~s}$ and fixed initial lead angle $\eta_{0}=40^{\circ}$ where the target moves with the ballistic inclination angle $0^{\circ}$ and speed of $20 \mathrm{~m} / \mathrm{s}$. In Figure 6(a), it is proved that the proposed acceleration instruction is suitable for attacking a target with constant speed by applying PIP concept. Under three desired impact time simulation conditions, the corresponding miss distances are $0.4194 \mathrm{~m}, 0.4774 \mathrm{~m}$, and $0.3833 \mathrm{~m}$, which implies that there is a better performance against moving targets in turn. It can be seen from Figure 6(b) that there is no obvious difference in the first 15 seconds for remaining attack time, and then, the difference among them gradually increases so as to meet the constraints of impact time. As is shown in Figures 6(c)$6(\mathrm{e})$, similar to case 2 , the proposed acceleration can satisfy both the constraints of desired impact time and FOV for moving target. Bedsides, since the calculation of the remaining time is essentially an estimation method, there is a certain error compared with the real remaining time. Thus, the calculation of the coordinates of the moving target will also accumulate errors. As a result, the sliding mode surface cannot converge to be zero like case 2. However, the errors of the three simulation conditions are $0.301 \mathrm{~s}, 0.132 \mathrm{~s}$, and $0.215 \mathrm{~s}$, respectively, which is within a reasonably acceptable range. In Figure 6(f), it can be observed by setting an appropriate impact time that it can improve the effectiveness of damage to a moving target to some extent.

\section{Conclusion}

In view of the antiship combat scenario, based on the equivalent sliding mode control method, a guidance law is put forward with both impact time and FOV constrained. An estimation of remaining flying time which is suitable for a large initial lead angle is adopted so as to allow more appli- cability in achievement of desired impact time. A sigmoid function is applied in the acceleration instruction to ensure that there is no signal chattering phenomenon. By using the proposed acceleration instruction, simulation results demonstrate that for a stationary target the missile can attack it accurately with FOV constrained under different limitations of initial lead angle and impact time. Besides, a cooperative attack schema is conducted in order to improve the attack efficiency for target. Moreover, PIP concept is utilized to achieve the attack of the target moving at a constant speed and simulation results show the effectives of the proposed acceleration instruction. The extension of the proposed guidance law to three-dimensional engagement and inclusion of impact angle criterion along with impact time constraint in the proposed is an important area for future extension.

\section{Nomenclature}

$R$ : Relative range between missile and target

$\gamma$ : LOS angle of missile

$a$ : Acceleration instruction normal to LOS of missile

$\eta$ : Lead angle of missile

$V$ : Speed of missile

$\theta$ : Flight path angle of missile.

\section{Data Availability}

All data in our paper are included within the article.

\section{Conflicts of Interest}

All the authors declare that they have no known competing financial interests or personal relationships that could have appeared to influence the work reported in this paper. 


\section{Acknowledgments}

The authors would like to appreciate the financial support from the National Natural Science Foundation of China (NSFC) (61603297) and the Natural Science Foundation of Shaanxi Province (2020JQ-219).

\section{References}

[1] S. R. Kumar and D. Ghose, "Impact time guidance for large heading errors using sliding mode control," IEEE Transactions on Aerospace and Electronic Systems, vol. 51, no. 4, pp. 31233138, 2015.

[2] A. Sinha and S. R. Kumar, "Supertwisting control-based cooperative salvo guidance using leader-follower approach," IEEE Transactions on Aerospace and Electronic Systems, vol. 56, no. 5, pp. 3556-3565, 2020.

[3] Byung Soo Kim, Jang Gyu Lee, and Hyung Seok Han, "Biased PNG law for impact with angular constraint," IEEE Transactions on Aerospace and Electronic Systems, vol. 34, no. 1, pp. 277-288, 1998.

[4] P. Zarchan, Tactical and strategic missile guidance, American Institute of Aeronautics and Astronautics, Inc, 2012.

[5] Min-Jea Tahk, Chang-Kyung Ryoo, and Hangju Cho, "Recursive time-to-go estimation for homing guidance missiles," IEEE Transactions on Aerospace and Electronic Systems, vol. 38, no. 1, pp. 13-24, 2002.

[6] S. Ghosh, D. Ghose, and S. Raha, "Three dimensional PN based impact angle control for higher speed nonmaneuvering targets," in In 2013 American control conferencepp. 31-36, Washington, DC, USA, 2013, June.

[7] S. Ghosh, D. Ghose, and S. Raha, "Unified time-to-go algorithms for proportional navigation class of guidance," Journal of Guidance, Control, and Dynamics, vol. 39, no. 6, pp. 11881205, 2016.

[8] C. K. Ryoo, H. Cho, and M. J. Tahk, "Optimal guidance laws with terminal impact angle constraint," Journal of Guidance, Control, and Dynamics, vol. 28, no. 4, pp. 724-732, 2005.

[9] T. H. Kim, C. H. Lee, I. S. Jeon, and M. J. Tahk, "Augmented polynomial guidance with impact time and angle constraints," IEEE Transactions on Aerospace and Electronic Systems, vol. 49, no. 4, pp. 2806-2817, 2013.

[10] M. Snyder, Z. Qu, R. Hull, and R. Prazenica, "Quad-segment polynomial trajectory guidance for impact-time control of precision-munition strike," IEEE Transactions on Aerospace and Electronic Systems, vol. 52, no. 6, pp. 3008-3023, 2016.

[11] G. A. Harrison, "Hybrid guidance law for approach angle and time-of-arrival control," Journal of Guidance, Control, and Dynamics, vol. 35, no. 4, pp. 1104-1114, 2012.

[12] Y. I. Lee, S. H. Kim, J. I. Lee, and M. J. Tahk, "Analytic solutions of generalized impact-angle-control guidance law for first-order lag system," Journal of Guidance, Control, and Dynamics, vol. 36, no. 1, pp. 96-112, 2013.

[13] C. H. Lee, T. H. Kim, and M. J. Tahk, "Interception angle control guidance using proportional navigation with error feedback," Journal of Guidance, Control, and Dynamics, vol. 36, no. 5, pp. 1556-1561, 2013.

[14] Chang-Kyung Ryoo, Hangju Cho, and Min-Jea Tahk, “Timeto-go weighted optimal guidance with impact angle constraints," IEEE Transactions on Control Systems Technology, vol. 14, no. 3, pp. 483-492, 2006.
[15] I. H. Whang and W. S. Ra, "Time-to-go estimation filter for anti-ship missile application," in 2008 SICE Annual Conference, pp. 247-250, Chofu, Japan, 2008, August.

[16] A. Ratnoo and D. Ghose, "State-dependent Riccati-equationbased guidance law for impact-angle-constrained trajectories," Journal of Guidance, Control, and Dynamics, vol. 32, no. 1, pp. 320-326, 2009.

[17] H. S. Shin, H. Cho, and A. Tsourdos, "Time-to-go estimation using guidance command history," IFAC Proceedings Volumes, vol. 44, no. 1, pp. 5531-5536, 2011.

[18] K. Chen, Y. Guo, S. C. Wang, and F. Xia, "A survey on guidance law with impact time constraint," in 2016 35th Chinese Control Conference (CCC), pp. 5711-5715, Chengdu, China, 2016, July.

[19] X. Wang, Y. Zhang, and H. Wu, "Distributed cooperative guidance of multiple anti-ship missiles with arbitrary impact angle constraint," Aerospace Science and Technology, vol. 46, pp. 299-311, 2015.

[20] Y. Zhang, X. Wang, and H. Wu, "A distributed cooperative guidance law for salvo attack of multiple anti-ship missiles," Chinese Journal of Aeronautics, vol. 28, no. 5, pp. 1438-1450, 2015.

[21] Y. Zhang, X. Wang, and H. Wu, "Impact time control guidance law with field of view constraint," Aerospace Science and Technology, vol. 39, pp. 361-369, 2014.

[22] D. Sang and M. J. Tahk, "Guidance law switching logic considering the seeker's field-of-view limits," Proceedings of the Institution of Mechanical Engineers, Part G: Journal of Aerospace Engineering, vol. 223, no. 8, pp. 1049-1058, 2009. 